SUPPORTING INFORMATION

\title{
Diffusion Dynamics of the Li atom on a model surface of amorphous carbon: A direct molecular orbital-molecular dynamics Study
}

\author{
Hiroto TACHIKAWA and Akira SHIMIZU \\ Department of Materials Chemistry, Faculty of Engineering, Hokkaido University, Sapporo \\ 060-8628, Japan
}

Potential energy curves for diffusion of Li atom between ring-over sites

The potential energy curves for diffusion of Li atom are given in Figure S1.

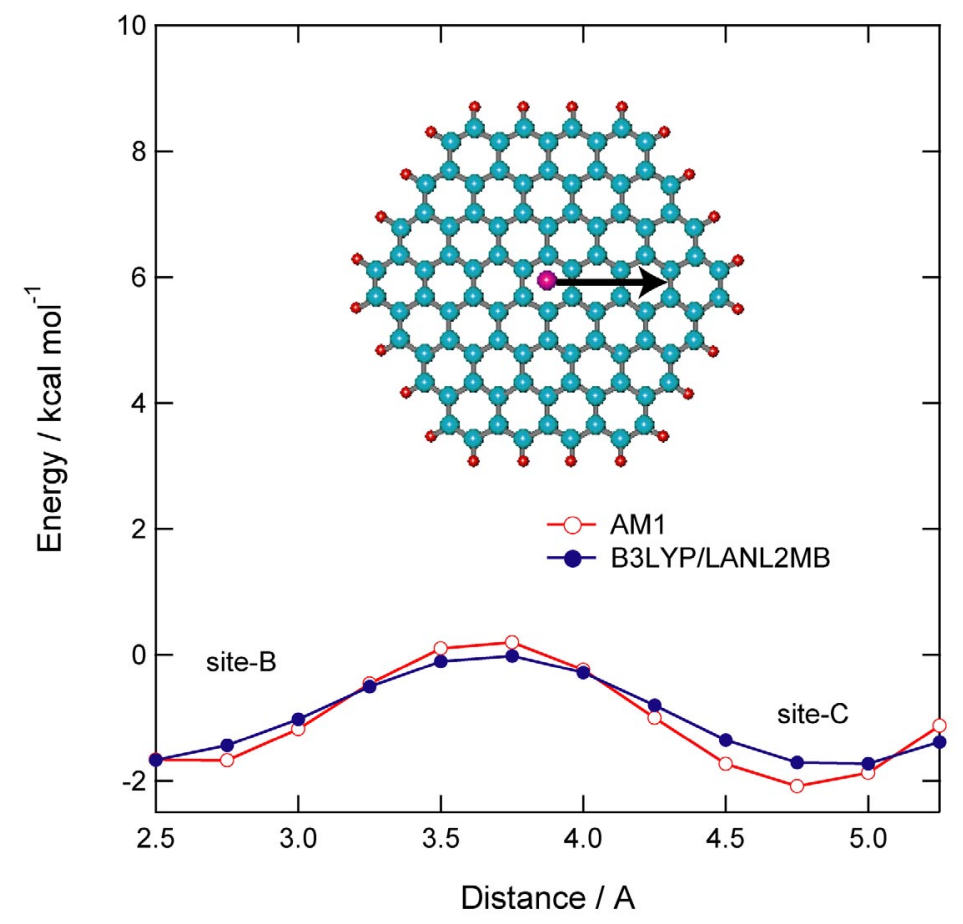

Figure S1. Potential energy curves for diffusion of $\mathrm{Li}$ atom between ring-over sites (B and $\mathrm{C})$ calculated by AM1 and B3LYP/LANL2MB. 